\title{
Polymer PCF Bragg grating sensors based on poly(methyl methacrylate) and TOPAS cyclic olefin copolymer
}

Johnson, lan P; Webb, David J; Kalli, Kyriacos; Yuan, Scott Wu; Stefani, Alessio; Nielsen, Kristian; Rasmussen, Henrik K.; Bang, Ole

\section{Published in:}

Proceedings of SPIE - The International Society for Optical Engineering

Link to article, DOI:

$10.1117 / 12.890090$

Publication date:

2011

Document Version

Publisher's PDF, also known as Version of record

Link back to DTU Orbit

Citation (APA):

Johnson, I. P., Webb, D. J., Kalli, K., Yuan, S. W., Stefani, A., Nielsen, K., Rasmussen, H. K., \& Bang, O. (2011). Polymer PCF Bragg grating sensors based on poly(methyl methacrylate) and TOPAS cyclic olefin copolymer. Proceedings of SPIE - The International Society for Optical Engineering, 8073, 80732V. https://doi.org/10.1117/12.890090

\section{General rights}

Copyright and moral rights for the publications made accessible in the public portal are retained by the authors and/or other copyright owners and it is a condition of accessing publications that users recognise and abide by the legal requirements associated with these rights.

- Users may download and print one copy of any publication from the public portal for the purpose of private study or research.

- You may not further distribute the material or use it for any profit-making activity or commercial gain

- You may freely distribute the URL identifying the publication in the public portal 


\title{
Polymer PCF Bragg grating sensors based on poly(methyl methacrylate) and TOPAS cyclic olefin copolymer
}

\author{
Ian P. Johnson ${ }^{\mathrm{a}}$, David J. Webb ${ }^{\mathrm{a}}$, Kyriacos Kalli ${ }^{\mathrm{b}}$, Wu Yuan ${ }^{\mathrm{c}}$, Alessio Stefani ${ }^{\mathrm{c}}$, Kristian Nielsen ${ }^{\mathrm{d}}$, \\ Henrik K. Rasmussen ${ }^{\mathrm{c}}$, Ole Bang ${ }^{\mathrm{C}}$. \\ a Photonics Research Group, Aston University, Aston Triangle, Birmingham. B4 7ET. UK. \\ ${ }^{\mathrm{b}}$ Nanophotonics Research Laboratory, Cyprus University of Technology, Cyprus. \\ c Tech Univ Denmark, Dept Photon Eng., DTU Fotonik, DK-2800 Lyngby, Denmark \\ d Tech Univ Denmark, Dept Mech Eng., DTU Mekanik, DK-2800 Lyngby, Denmark
}

\begin{abstract}
Fibre Bragg grating (FBG) sensors have been fabricated in polymer photonic crystal fibre (PCF). Results are presented using two different types of polymer optical fibre (POF); first multimode PCF with a core diameter of $50 \mu \mathrm{m}$ based on poly(methyl methacrylate) (PMMA) and second, endlessly single mode PCF with a core diameter of $6 \mu \mathrm{m}$ based on TOPAS cyclic olefin copolymer. Bragg grating inscription was achieved using a 30mW continuous wave 325nm helium cadmium laser. Both TOPAS and PMMA fibre have a large attenuation of around $1 \mathrm{~dB} / \mathrm{cm}$ in the $1550 \mathrm{~nm}$ spectral region, limiting fibre lengths to no longer than $10 \mathrm{~cm}$. However, both have improved attenuation of under $10 \mathrm{~dB} / \mathrm{m}$ in the $800 \mathrm{~nm}$ spectral region, thus allowing for fibre lengths to be much longer. The focus of current research is to utilise the increased fibre length, widening the range of sensor applications. The Bragg wavelength shift of a grating fabricated in PMMA fibre at $827 \mathrm{~nm}$ has been monitored whilst the POF is thermally annealed at $80{ }^{\circ} \mathrm{C}$ for 7 hours. The large length of POF enables real time monitoring of the grating, which demonstrates a permanent negative Bragg wavelength shift of $24 \mathrm{~nm}$ during the 7 hours. This creates the possibility to manufacture multiplexed Bragg sensors in POF using a single phase mask in the UV inscription manufacturing. TOPAS holds certain advantages over PMMA including a much lower affinity for water, this should allow for the elimination of cross-sensitivity to humidity when monitoring temperature changes or axial strain, which is a significant concern when using PMMA fibre.
\end{abstract}

\section{INTRODUCTION}

Over roughly the last decade the mature technology of FBG sensor fabrication in step index silica fibre has steadily been transferred to polymer optical fibre (POF), with the first FBG sensor fabricated in step index POF in the late $1990 \mathrm{~s}^{[1]}$. Following this, FBG sensors were fabricated in polymer few-moded $\mathrm{PCF}^{[2]}$ in an attempt to take advantage of the POF characteristics over silica fibre. These advantages include that polymers are clinically acceptable within the human body during surgical investigation, due to the fact that POFs are non-brittle and flexible, potentially lending them nicely to invivo sensing applications ${ }^{[3]}$. Additionally polymers can have a Young's modulus which is 25 times smaller than of silica $^{[4]}$, which in turn permits the monitoring of compliant structures and a high failure strain has allowed demonstrations of FBG sensor tuning in step index POF by using recoverable strains as large as $13 \%{ }^{[5]}$.

In this paper polymer FBG sensor fabrication is discussed in both multimode PCF, which is based on poly(methyl methacrylate) (PMMA), and in endlessly single mode PCF formed from TOPAS cyclic olefin copolymer. Multimode PCF is of interest to future research as it has the potential of offering a more economical solution to POF sensing. The use of multimode PCF offers possibilities of easier connectorisation to silica fibre with the potential of embedding the PCF in a ferrule connector as the larger core provides better tolerance to slight misalignments. Furthermore, multimode PCF allows the use of cheaper broad area emitting light sources when compared to single transverse mode light sources. Broadly speaking polymer PCF compared to silica optical fibre is simpler to manufacture and lends itself to possible chemical modification using organic techniques. An inconvenience of FBG sensors fabricated in PMMA PCF is the influence of absorbed water. This results in a cross-sensitivity to relative humidity when these devices are employed in applications such as axial strain and temperature sensing. TOPAS however has the potential of eliminating this cross-

Optical Sensors 2011; and Photonic Crystal Fibers V, edited by Francesco Baldini, Jiri Homola, Robert A. Lieberman, Kyriacos Kalli, Proc. of SPIE Vol. 8073, 80732V · () 2011 SPIE · CCC code: 0277-786X/11/\$18 · doi: 10.1117/12.890090 
sensitivity to relative humidity as it has a much lower water affinity when compared to PMMA. Moreover TOPAS has the potential to be employed in novel bio-sensing applications due to the requirement of linker molecules when attaching bio-sensing layers, which allows specific sensing areas to be defined ${ }^{[6]}$.

This paper reports on the fabrication of FBG sensors in both PMMA and TOPAS based PCF within the 1550nm spectral region. Building upon this is the fabrication of FBG sensors in the multimode PMMA PCF within the 800nm spectral region, taking advantage of the lower attenuation at these wavelengths of less than $10 \mathrm{~dB} / \mathrm{m}^{[7]}$. The fabrication of FBG sensors at these lower wavelengths has allowed larger lengths of the PMMA multimode PCF to be used. This has been utilised to monitor the real-time Bragg wavelength shift due to thermally annealing the PCF at $80^{\circ} \mathrm{C}$ for 7 hours, which induced a permanent negative Bragg wavelength shift of 22nm.

The permanent blue wavelength shifts experienced have been exploited to manufacture the first documented three FBG wavelength division multiplexed (WDM) sensors within the $800 \mathrm{~nm}$ spectral region whilst only using a single phase mask during fabrication. Additional three FBG WDM sensors were fabricated using two separate phase masks and employing thermal annealing tuning to produce a multiplexed sensor designed to reduce the possibility of crosstalk in high-strain sensing applications.

\section{FIBRE BRAGG GRATING FABRICATION}

Polymer FBGs have been fabricated in two types of polymer PCF. The first of these fibres is a multimode polymer PCF, as shown in Figure 1a. This fibre has an outer diameter of $150 \mu \mathrm{m}$ and a $50 \mu \mathrm{m}$ core. The core is created by three rings of holes; the higher refractive core is enclosed within a lower effective refractive index region created by a total of 72 holes, thus providing index guiding in the core. The multimode PCF is made purely from PMMA and is acquired from Kiriama Pty. Ltd. Sydney, Australia, a detailed description of the manufacturing process of the multimode PCF is documented by Barton et al without the sleeving process detailed ${ }^{[8]}$.

The second POF to be used is an endlessly single mode polymer PCF shown in Figure 1b. This fibre has an outer diameter of $270 \mu \mathrm{m}$ and a core diameter of $6 \mu \mathrm{m}$. On this occasion index guiding is provided in the higher refractive index core by having two rings of holes providing a lower effective refractive index around the core. A total of 18 holes are used each with a diameter of $2 \mu \mathrm{m}$. The fibre is manufactured at the Technical University of Denmark; it is manufactured purely from TOPAS cyclic olefin copolymer and is formed from a solid cylindrical preform of diameter $6 \mathrm{~cm}$ and drawn into fibre in a two stage process.
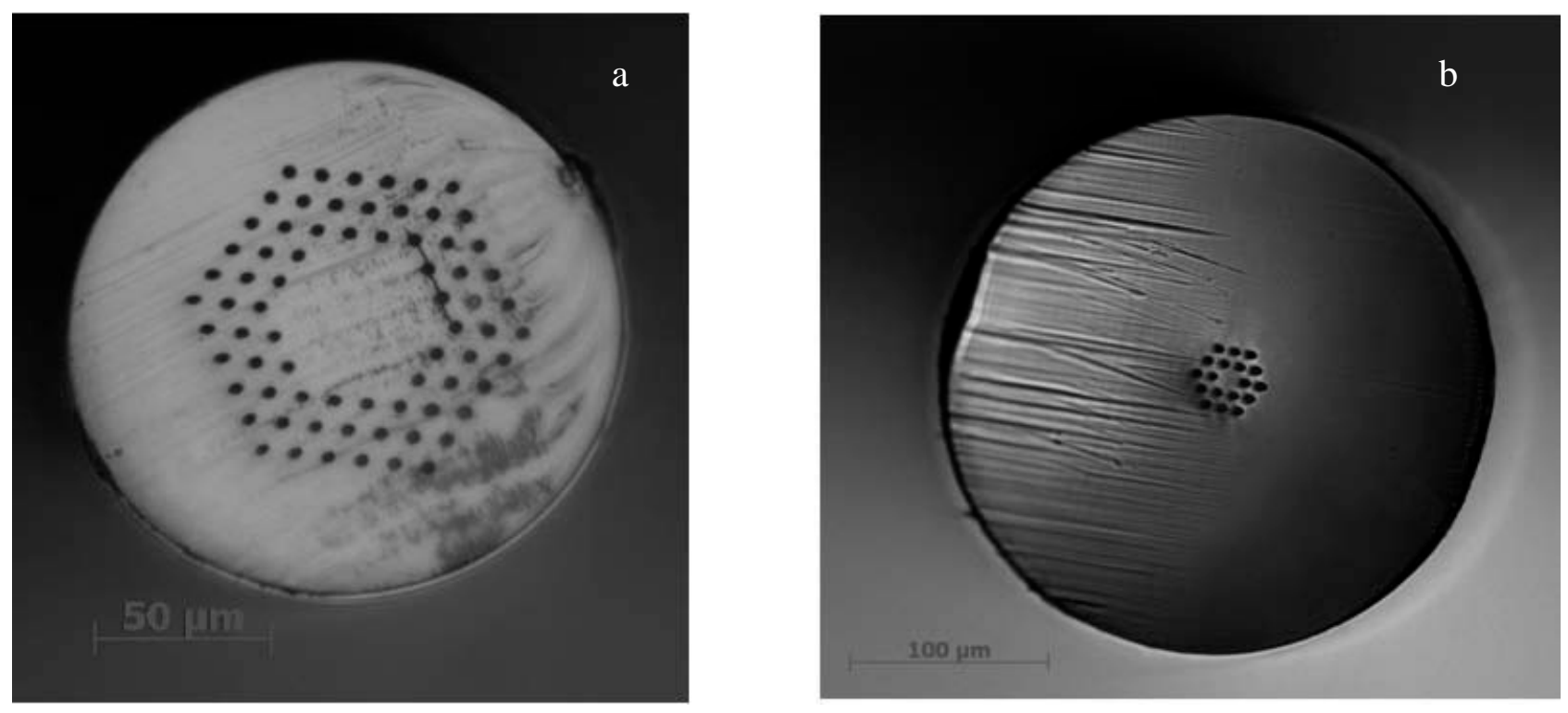

Figure 1 Microscope images of a: multimode PMMA PCF. b: Endlessly single mode TOPAS PCF 
Bragg grating fabrication in both types of fibre is carried out using the same phase mask technique. A helium-cadmium (He-Cd) continuous wave laser (Kimmon IK3301R-G), emitting at 325nm with an output of $30 \mathrm{~mW}$ is used for all manufactured polymer FBGs. The laser beam is focussed vertically downwards into the POF using a cylindrical lens with a focal length of $10 \mathrm{~cm}$, oriented such that the focal line lies along the fibre axis. An optimised phase mask for $325 \mathrm{~nm}$ exposure is rested directly on top of the fibre, which the UV beam passes through, exposing a periodic structure into the core of the fibre. This is a minimal fabrication setup, however the essence of the setup is to keep the POF immobile during the entire time period of the fabrication since fabricating a Bragg grating in POF can take anything from 40 to 90 minutes. To eliminate the POF from drooping or sagging, which can be exaggerated by the heat generated within the fibre during the fabrication, the PCF is mounted onto a V-groove plate along its entire length and taped into position using polyimide tape. This in turn enables a phase mask to be supported directly on top of the PCF and v-groove plate. To ensure stability of the POF, the fibre is not translated and as the UV beam is stationary the Bragg grating length is limited to the width of the laser which in this case is $1.8 \mathrm{~mm}$.

As discussed the main aim of the fabrication setup is to ensure the PCF is immobile during fabrication thus guaranteeing the UV beam is aligned to the core of the PCF fibre during the entire fabrication time period. The UV beam is initially

aligned to the core of the PCF by monitoring the back scattering of the UV beam reflected back from the PCF outer surface. Using this method has proved successful in the past and with experience this technique can become very reliable when inscribing Bragg gratings into either of the two types of polymer PCF.

The Bragg gratings fabricated in these two types of polymer PCF are monitored in reflection, here an arm of a coupler is butt coupled to the PCF on a translation stage. A small amount of index matching gel is used to reduce the Fresnel reflections from the coupler arm; care should also be taken not to use too much gel or it will travel along the hole structure of the PCF and effect the index guiding conditions. Either a single or multimode coupler is used depending on which PCF is being used, in the case of the multimode PCF the interrogating light source is first passed through $20 \mathrm{~m}$ of silica multimode step index fibre $(50 / 125 \mu \mathrm{m})$ wound around a reel so that the light incident on the grating approaches an equilibrium modal distribution. In all monitoring of FGB fabrication and sensor characterisation, the reflected Bragg response has been captured on an optical spectrum analyser (HP 70004A). Two interrogating light sources are used to monitor the polymer FBGs depending purely on which spectral region the Bragg grating has been fabricated in. In the case of the 1500nm spectral region a broadband light source has been used (Thorlabs, ASE-FL7002-C4) and in the case of the 800nm spectral region an Amonics super wideband short wavelength light source (ASLD-CWDM-3-B-FA).

\section{BRAGG GRATINGS IN POLYMER PCF}

Polymer FBGs have previously been reported in the multimode PMMA PCF ${ }^{[9]}$. Bragg gratings were inscribed for approximately 55minutes, which resulted in a response with a Bragg wavelength of 1562nm when using a phase mask with a period of $1057.2 \mathrm{~nm}$. The typical reflection spectrum of the Bragg response has a signal-to-noise ratio of 20dB and a FWHM of $4 \mathrm{~nm}$ when observed on an OSA using a bandwidth of $0.5 \mathrm{~nm}$. Polymer FBG sensor fabrication in the multimode polymer PCF has proved very consistent with an approximate success rate at FBG inscription of 95\%. This fibre is often used to fault-find the UV laser inscription setup due to its robustness and large core diameter.

Fibre Bragg grating inscription has now also been proven to be very successful in the TOPAS PCF with an inscription success rate approaching that of the multimode PMMA PCF. The first successful FBG fabrication in TOPAS was documented in early $2011^{[10]}$. Here a Bragg grating was inscribed into the TOPAS PCF in 45 minutes using a phase mask with a period of 1034.2nm, resulting in a response with a Bragg wavelength of 1567.9nm and a bandwidth (FWHM) of 0.75nm. Since then FBGs have been consistently fabricated in the TOPAS PCF. As can be seen in Figure 2a, the typical Bragg wavelength is $1568.35 \mathrm{~nm}$ with a FWHM of $1.13 \mathrm{~nm}$ when using the mask previously used with a period of 1034.2nm. Figure 2b demonstrates it takes around 40 minutes to fabricate a FBG sensor in the TOPAS PCF, from experience a saturation level will be reached after this time period. The slight decrease in the reflected peak power in the first ten minutes is due to fluctuations in the noise level being monitored rather than a reflected optical signal. 

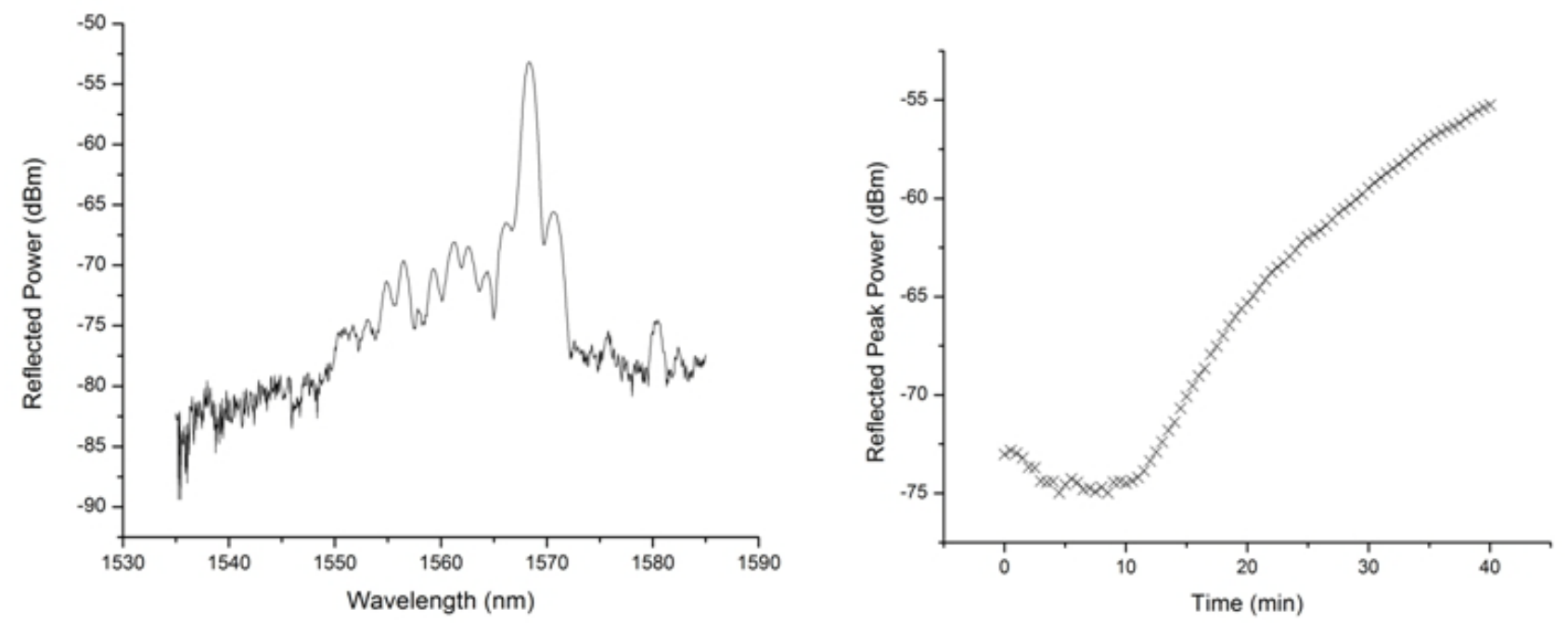

Figure 2 a: reflection spectrum of a FBG fabricated in TOPAS PCF, b: growth curve of the reflected peak signal

\section{LOW ATTENUATION}

Fibre Bragg grating sensors fabricated thus far either in the PMMA multimode PCF or the endlessly single mode TOPAS PCF have all operated within the C-Band spectral region. Sensors which function at these wavelengths are limited to lengths of no longer than $10 \mathrm{~cm}$ due to attenuations experienced of around $1 \mathrm{~dB} / \mathrm{cm}$ at $1550 \mathrm{~nm}^{[7]}$. Although an optical adhesive splicing technique to silica fibre has been developed to allow POF grating sensors to move away from the optical bench and into application specific setups, sensors are located within a few centimetres of the fragile and bulky splices, hence limiting the range of applications ${ }^{[9,11]}$. Therefore research has been carried out working towards the inscription of FBG sensors in both the PMMA and TOPAS PCFs in the $800 \mathrm{~nm}$ spectral regions. Here the fibre has a much smaller attenuation of less than $10 \mathrm{~dB} / \mathrm{m}$, this will therefore allow sensor lengths of $1 \mathrm{~m}$, thereby greatly widening the applications that can be addressed ${ }^{[7]}$.

The first FBG sensor in the PMMA multimode PCF reported to take advantage of the lower attenuation at the 800nm spectral region was documented by Johnson et $a l^{[12]}$. Here a FBG sensor was fabricated in the PMMA multimode PCF using a phase mask with a period of $557.20 \mathrm{~nm}$. The resulting reflected Bragg response had a Bragg wavelength of $827 \mathrm{~nm}$ and a bandwidth of $2.45 \mathrm{~nm}$ (FWHM) when using an OSA with a $0.5 \mathrm{~nm}$ bandwidth resolution. A signal-to-noise ratio of around $12 \mathrm{~dB}$ was achieved after 2hours when a saturation level was reached.

Recent work has been aimed towards the reliable fabrication of FBG sensors in the PMMA multimode PCF within the $800 \mathrm{~nm}$ spectral region. It is now considered that the success rate of the fabrication of FBG sensors in this fibre with a Bragg response in the $800 \mathrm{~nm}$ spectral region is greater than 95\%. Figure 3 is another example of a FBG sensor fabricated in the PMMA multimode PCF. As can be seen in Figure 3a, when using the same mask with a period of 557.50nm as Johnson et a ${ }^{[12]}$ a typical FBG sensor can be fabricated with a Bragg response with a wavelength of $827.86 \mathrm{~nm}$ and a bandwidth of $0.69 \mathrm{~nm}$ (FWHM) when captured with an OSA with a $0.5 \mathrm{~nm}$ bandwidth resolution. Sensors fabricated at this specific spectral wavelength in the PMMA multimode PCF are now consistently fabricated within one hour before a saturation level of the reflected power is reached, as demonstrated in Figure 3b where a saturation level is reached after 50 minutes. This is a significant improvement on the initially presented results ${ }^{[12]}$ and has been a result of further experience at working at this lower spectral region. 

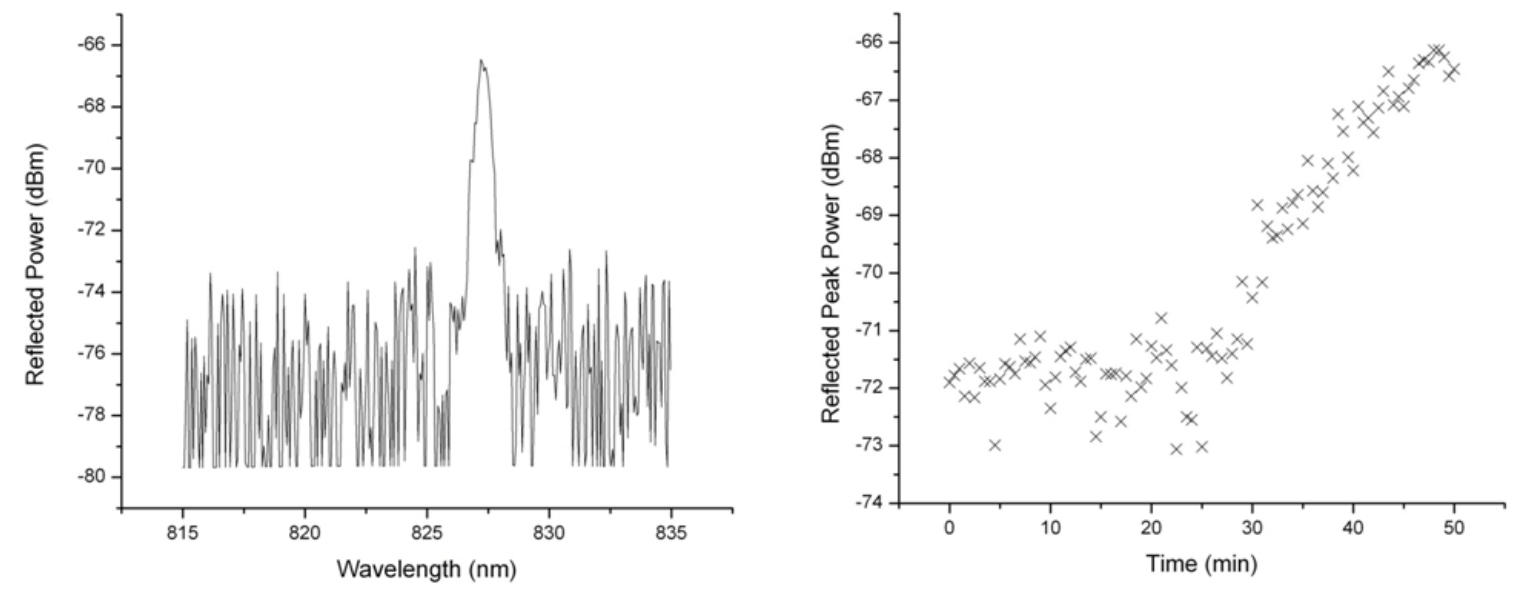

Figure 3 a: reflection spectrum of a FBG sensor fabricate in multimode PMMA PCF with a Bragg wavelength of 827nm, b: growth curve of the reflected peak signal

Additionally, FBG sensors can be fabricated with a Bragg wavelength of $860.65 \mathrm{~nm}$ as can be seen in Figure 4a; here the sensor has been inscribed using a phase mask with a period of 580nm. The Bragg response has a similar bandwidth of $0.81 \mathrm{~nm}$ to FBG sensors fabricated at $827 \mathrm{~nm}$ and once more Figure $4 \mathrm{~b}$ demonstrates a saturation level of the reflected power being reached after 45 minutes.
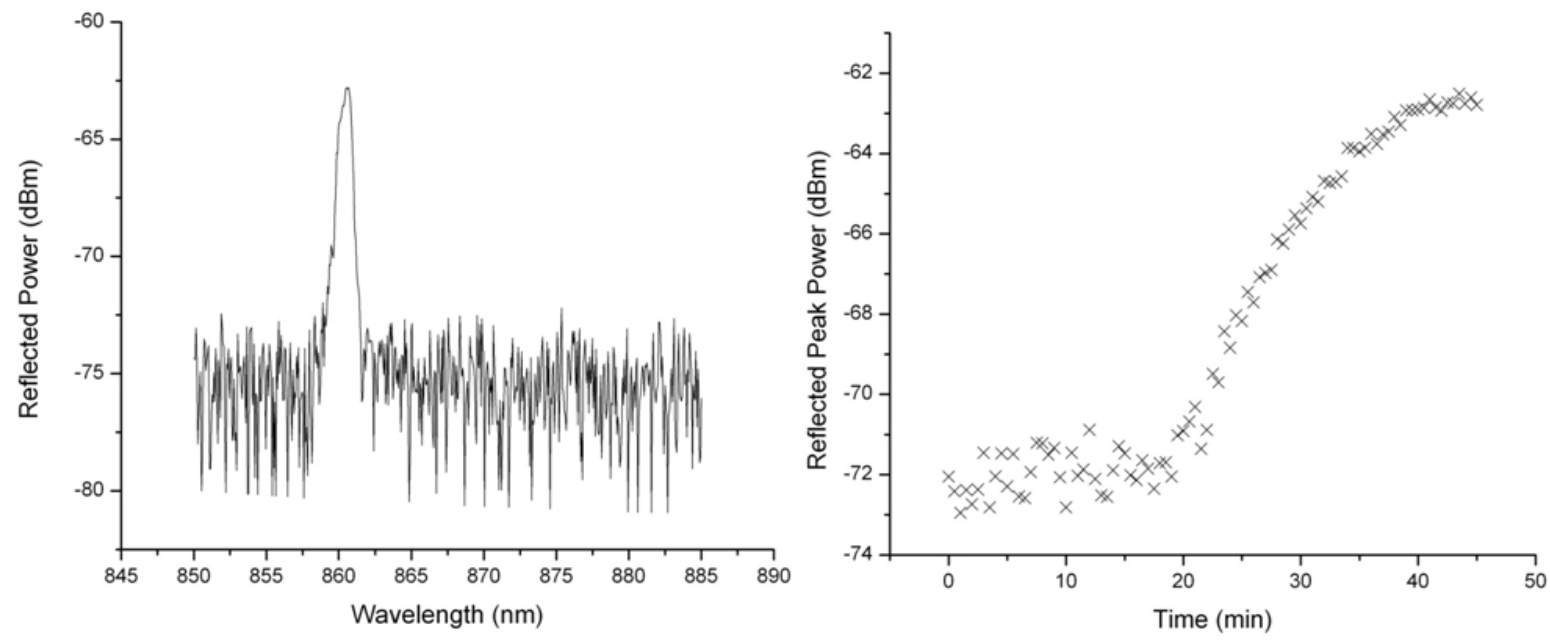

Figure 4 a: reflection spectrum of FBG sensor fabricated in multimode PMMA PCF with a Bragg wavelength of 680nm, b: growth curve of the reflected peak signal 


\section{THERMAL ANNEALING}

The possibility of using longer lengths of POF is now realised when fabricating FBG sensors within the 800nm spectral region, this allows for a wider range of applications for the FBG sensors. As the attenuation is less than $10 \mathrm{~dB} / \mathrm{m}$ in the 800nm spectral region the FBG sensor fabricated with a Bragg wavelength of 860.65nm shown in Figure 3 has been inscribed into the PMMA multimode PCF with a length of $20 \mathrm{~cm}$ from the inscription point to the optical adhesive splice to a silica pigtail. This permits the possibility of lowering the Bragg sensor into an oven pre-heated to $80^{\circ} \mathrm{C}$ whilst protecting the adhesive splice, which is susceptible to failure at temperatures above $40^{\circ} \mathrm{C}$. This in turn allows for realtime monitoring of the effects on the Bragg response when thermally annealing the FBG sensor at $80^{\circ} \mathrm{C}$ for seven hours. The monitored Bragg wavelength shift with time can be seen in Figure 5. The sensor was lowered into the oven after 30 minutes of monitoring the Bragg wavelength, after seven hours of monitoring a permanent blue wavelength of 22nm was observed.

As just seen, thermally annealing a FBG sensor inscribed in polymer PCF induces a permanent blue wavelength in the reflected Bragg response ${ }^{[13]}$. The origin of this shift lies with the fact that the tension applied to the polymer when drawing into fibre results in the partial alignment of the molecular chains along the axis of the fibre. As the polymer PCF is heated a temperature will be reached where the molecular chains start to relax from this alignment causing the fibre to shrink along its length. The temperature at which the fibre starts to shrink along its length is believed to be related to the thermal history of the polymer together with the drawing specifications ${ }^{[14]}$.

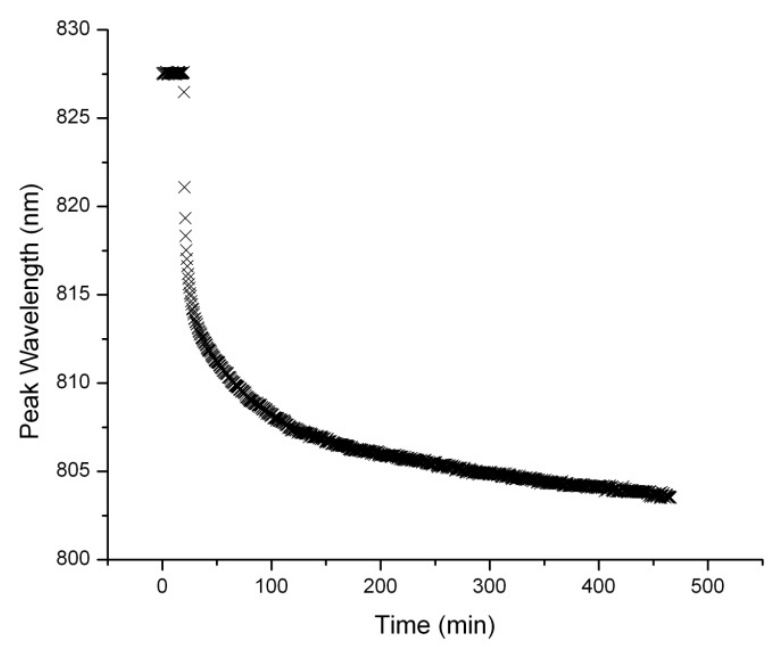

Figure 5 Real-time monitoring of the thermal annealing of a FBG sensor fabricated in multimode PMMA PCF with a Bragg length of $860 \mathrm{~nm}$. Thermal annealing was at $80^{\circ} \mathrm{C}$ for 7 hours.

\section{WAVELENGTH DIVISION MULTILEXED SENSORS}

The ability to be able to permanently thermally tune the Bragg response of a sensor fabricated in PMMA multimode PCF can be advantageous. If a specific narrow band light source is required to interrogate a sensor, such as a vertical cavity surface emitting laser (VCSEL), then possibly a specific phase mask would have to be purchased to fabricate a sensor with a matching Bragg wavelength. With the cost of phase mask manufacture relatively expensive a more economical option may be to thermally tune a FBG sensor fabricated using an existing phase mask.

Thermally annealing a FBG sensor in polymer PCF can also be utilised to manufacture WDM FBG sensors. Two examples are given demonstrating the fabrication WDM sensors in the PMMA multimode PCF within the 800nm spectral region. The first example of a fabricated WDM sensor is shown in Figure 6a, here the entire WDM sensor is fabricated with a single phase mask with a period of 557.50nm. Firstly the fibre is thermally pre-annealed for ten minutes 
at $80^{\circ} \mathrm{C}$ to escape the initial rapid wavelength shift. Grating 1 is then inscribed for 90 minutes with a Bragg wavelength of $827 \mathrm{~nm}$, this grating is then thermally annealed for 30 minutes at $80^{\circ} \mathrm{C}$ resulting in a permanent blue wavelength shift of $3.5 \mathrm{~nm}$. Grating 2 is then fabricated at $827 \mathrm{~nm}$ for 90 minutes and the PCF is then annealed for 2 hours, this resulted in grating 1 experiencing an additional permanent blue Bragg wavelength shift of $1 \mathrm{~nm}$ and grating 2 a blue Bragg wavelength shift of $3.2 \mathrm{~nm}$. Finally the third grating is fabricated again for 90 minutes with a Bragg wavelength of $827 \mathrm{~nm}$. Each FBG sensor was separated by $10 \mathrm{~mm}$ along the fibre axis. Optimising the thermal recipe to create three equally spaced FBGs for a WDM sensor using a single phase mask proved to be a difficult operation and thus there is a significant chance of crosstalk when using this WDM sensor in any application.

The second example of a WDM sensor is shown in Figure 6b, this PCF sensor has been fabricated using two phase masks. Firstly a FBG sensor is fabricated for 90 minutes resulting in a Bragg response at 860nm, the PMMA multimode PCF is then thermally annealed for 24 hours at $70^{\circ} \mathrm{C}$. The temperature was decreased by $10^{\circ} \mathrm{C}$ from the previous experiment so to safeguard the polymer PCF, with a concession made for the annealing length increased to 24 hours. The 24 hour thermal annealing resulted in a blue Bragg wavelength shift of $15.87 \mathrm{~nm}$, giving a permanent Bragg wavelength of $844.13 \mathrm{~nm}$ for FBG 1. Grating 2 was then fabricated again with the $580 \mathrm{~nm}$ period phase mask for a duration of 45 minutes, resulting in a second grating with a Bragg wavelength of $860.66 \mathrm{~nm}$. And finally a third grating was fabricated using a different phase mask with a smaller period of 557.50nm for a duration of 50 minutes. This resulted in a Bragg response at $827.20 \mathrm{~nm}$. The gratings were separated by $10 \mathrm{~mm}$ along the fibre axis. The three separate FBG sensors had Bragg wavelengths separated by at least $15 \mathrm{~nm}$ along the spectral region, thus decreasing the possibilities of crosstalk when employing this WDM sensor in high strain monitoring applications.
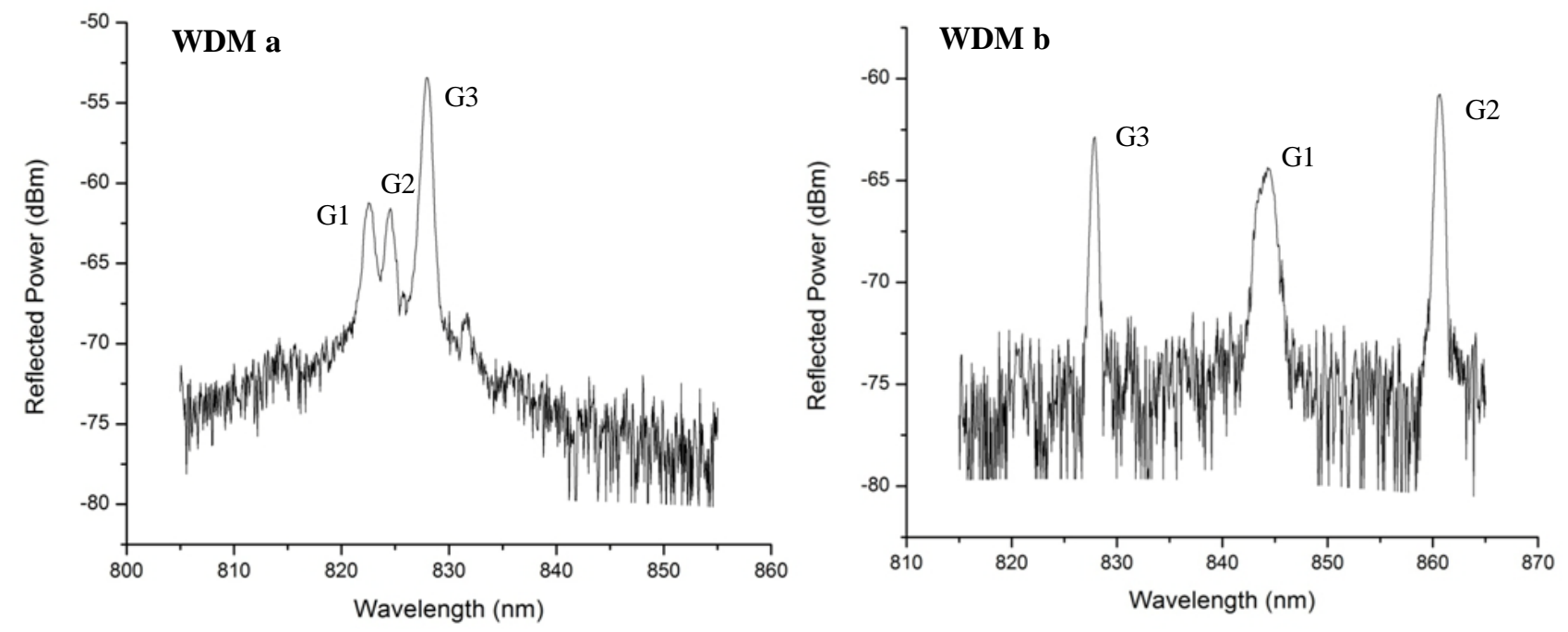

Figure 6 a: WDM sensor using a single phase mask and utilising thermal annealing, b: WDM sensor using two spate phase masks and a single thermal annealing stage.

\section{CONCLUSION}

It has been demonstrated that FBG sensors can be consistently fabricated in both PMMA and TOPAS PCF within the 1550nm spectral region. Moreover FBG sensors can now also be fabricated in multimode PMMA PCF within the 800nm spectral region with sensors fabricated with Bragg wavelengths at both $827 \mathrm{~nm}$ and $860 \mathrm{~nm}$. FBG sensor fabrication in the TOPAS PCF can also be achieved at lower attenuation wavelengths within the $800 \mathrm{~nm}$ spectral region using the same fabrication techniques. Manufacturing FBG sensors within the 800nm band in the PMMA multimode PCF has allowed the real-time monitoring of the thermal annealing of the sensor at $80^{\circ} \mathrm{C}$ for 7 hours. This results in the relaxation of the polymer molecular chains from the fibre axis orientation, causing the PCF to shrink. This gives an end outcome of a permanent negative Bragg wavelength shift of 22nm within the reflected spectrum. The thermal tuning of the multimode 
PMMA PCF allows the possibility to tune to a specific interrogating light source, providing a more economical choice when designing a sensing setup than purchasing multiple phase masks. Furthermore, thermal tuning creates the possibility of fabricating WDM sensors, which has been demonstrated by manufacturing the first three FBG WDM sensor in POF within the 800nm spectral region whilst only using a single phase mask for the three FBG inscriptions.

\section{ACKNOWLEDGEMENTS}

The authors would like to acknowledge the financial support of the European Commission via the FP7 project PHOtonic Skins For Optical Sensing (PHOSFOS).

\section{REFERENCES}

[1] Z. Xiong, et al., "Highly tunable Bragg gratings in single-mode polymer optical fibers," Ieee Photonics Technology Letters, vol. 11, pp. 352-354, Mar 1999.

[2] H. Dobb, et al., "Continuous wave ultraviolet light-induced fiber Bragg gratings in few- and single-mode microstructured polymer optical fibers," Optics Letters, vol. 30, pp. 3296-3298, 2005.

[3] F. Baldini and A. G. Mignani, "Optical-fiber medical sensors," Mrs Bulletin, vol. 27, pp. 383-387, May 2002.

[4] M. Silva-Lopez, et al., "Strain and temperature sensitivity of a single-mode polymer optical fiber," Optics Letters, vol. 30, pp. 3129-3131, Dec 2005.

[5] H. B. Liu, et al., "Strain and temperature sensor using a combination of polymer and silica fibre Bragg gratings," Optics Communications, vol. 219, pp. 139-142, Apr 2003.

[6] G. Emiliyanov, et al., "Localized biosensing with Topas microstructured polymer optical fiber," Optics Letters, vol. 32, pp. 460-462, Mar 2007.

[7] H. P. A. van den Boom, et al., "High-capacity transmission over polymer optical fiber," Ieee Journal of Selected Topics in Quantum Electronics, vol. 7, pp. 461-470, May-Jun 2001.

[8] G. Barton, et al., "Fabrication of microstructured polymer optical fibres," Optical Fiber Technology, vol. 10, pp. 325-335, Oct 2004.

[9] I. P. Johnson, et al., "Multiplexed FBG sensor recorded in multimode microstructured polymer optical fibre," 2010, p. 77140D.

[10] I. P. Johnson, et al., "Optical fibre Bragg grating recorded in TOPAS cyclic olefin copolymer," Electronics Letters, vol. 47, pp. 271-+, 2011.

[11] C. C. Ye, et al., "Applications of polymer optical fibre grating sensors to condition monitoring of textiles - art. no. 012020," in Sensors \& Their Applications Xv. vol. 178, A. T. Augousti and G. McConnell, Eds., ed Bristol: Iop Publishing Ltd, 2009, pp. 12020-12020.

[12] I. P. Johnson, et al., "827 nm Bragg grating sensor in multimode microstructured polymer optical fibre," Electronics Letters, vol. 46, pp. 1217-U74, Aug 2010.

[13] K. E. Carroll, et al., "Thermal response of Bragg gratings in PMMA microstructured optical fibers," Optics Express, vol. 15, pp. 8844-8850, Jul 2007.

[14] T. Ishigure, et al., "Graded-index plastic optical fiber with high mechanical properties enabling easy network installations. I," Journal of Applied Polymer Science, vol. 91, pp. 404-409, Jan 2004. 